\title{
Intelligent Proportional Differential Neural Network Control for Unknown Nonlinear System
}

\author{
Haoping WANG ${ }^{1}$, Shanzhi LI ${ }^{1}$, Yang TIAN ${ }^{1, *}$, Abdel AITOUCHE ${ }^{2}$ \\ ${ }^{1}$ Automation School, \\ Sino-French International Joint Laboratory of Automatic Control and Signal Processing (LaFCAS), \\ Nanjing University of Science\& Technology (NUST), \\ Nanjing 210094, China; \\ ${ }^{2}$ CRIStAL UMR CNRS 9189 , \\ Hautes etudes d'ingenieur HEI-Lille, \\ Lille,59046, France, \\ tianyang@njust.edu.cn (*corresponding author)
}

\begin{abstract}
This paper presents an intelligent proportion-differential neural network (iPDNN) controller for unknown nonlinear systems. This controller is based on the intelligent proportion integration differentiation (iPID) controller. In an iPID controller system, a unknown nonlinear SISO system is regarded as an ultra-local two-order or one-order model and a lumped unknown dynamics (LUD) disturbance which contains the high-term and parametric uncertainties by the differential algebra and estimation method online. However, its performance of an iPID control depends on the precision and rapidity for estimating the LUD disturbance. Besides, it also influences the parameter in the ultra-local model. In order to compensate the estimation error of LUD disturbance, we put forward an extra radial basis function (RBF) neural network observer to estimate it. This extra observer cannot only ensure to acquire the estimation error rapidly, but also has an ability of self-learning. In addition, this iPDNN method can ensure the closed-loop system stability under the Lyapunov stability theory. Finally, in order to demonstrate its performance, an inverted pendulum plant has been applied and the results indicate this method is of efficiency.
\end{abstract}

Keywords: adaptive control; mode- free control; PID.

\section{Introduction}

As a classical algorithm, adaptive control has been applied in many types of industrial systems including nonlinear and time-vary systems and also attracts many scholars' attentions. Because of the advantage of selfadaptation, adaptive controller can tolerate the uncertainties and external disturbances in closed-loop system [7, 11, 19, 21]. In the adaptive control system, a common method for dealing with uncertainties including the parameters or structure is to design an adaptive parameter estimates algorithm or an auxiliary controller $[3,4,14]$. A nonlinear system is difficult to use a mathematical equation for modelling because of the lack of knowledge of plant or disturbances $[9,10]$.

As for the issues, many research results have been put forward for applying a range of different perspectives. Their methods can be classified into two types according to the approximation form. The first type is called a direct approximation method such as model reference adaptive control (MRAC) [1, 15], which is used as a reference model to obtain the disturbance immediately. In this type of system, by comparing with the nominal model or reference model, the disturbance can be acquired directly. The other type used an indirect method $[2,8,26]$. In this type of system, it requires knowing about the information of structure and some parameters. Its performance depends on the model parameters.

Recently, as a new type of adaptive control, model-free control and its corresponding intelligent proportion integration differentiation (iPID) controller which has been applied successfully to the real-time systems, such as DC-DC converters [17], vehicle control [16], agricultural greenhouses [13], High pressure common rail injection system [22] etc., was firstly introduced in the references [5]. It requires only the systems input gain, and the output measurements. The critical issue for this kind model free control is resided on how to obtain and compensate rapidly the lumped unknown dynamics (LUD) which it covers the high-order term, uncertain parameters, external and or internal perturbations.-Reference [17] puts forward an algebraic identification technique to estimate the LUD. In reference [20], a Savitzky-Golay filter is proposed to obtain the LUD for a servo system. However, these methods regard the gain of input as a 
known constant and ignore its parameter-vary. In addition, its performance depends on the quality of the observer which requires the ability of rapidity and reliability. Moreover, these methods cannot guarantee the stability in closed-looped system $[12,16]$.

Considering these factors, an extra sliding model control is adopted into the model free control for compensating the estimation error of LUD [18]. However, the gain of input is also neglected, which stems from the ultra-local model. Thus in this paper, a radial basis function neural network is introduced to make up for the estimation error of the LUD. The referred neural network has an ability of universal approximation and been applied to a nonlinear system for estimation LUD. Combining the advantage of neural network, the novel model-free control strategy which is called as an intelligent Proportion Differential Neural Network (iPDNN) controller ensures not only the stability, but also has a high efficiency for trajectory tracking performance.

The paper is organized as follows. In section 2, the problem of a classical model free control is introduced. In section 3, an improved model free iPDNN control is proposed while in section 4 , to validate the proposed method, an inverted pendulum numerical system is implemented. Finally, some conclusion remarks are given in section 5 .

\section{Problem Statement}

For a general SISO unknown nonlinear system described in [18], its corresponding ultra-local model can be denoted approximately as follows

$$
y^{n}(t)=F(t)+\alpha u(t)
$$

where $n$ is the derivative order of the system output, $\alpha$ is an input gain, $u(t)$ is the control input, $y(t)$ is the system output, the integer $n$ is usually selected as 1 or 2 , and $F(t)$ is treated as an LUD, which contains the high-order term, the unknown parts of a practical system, measuring noise or various disturbances.

According to [6], the proposed iPIDis defined as

$$
\begin{aligned}
u(t) & =\frac{1}{\alpha}\left(k_{p} e(t)+k_{i} \int e(t) d t+\right. \\
& \left.+k_{d} \dot{e}(t)+\ddot{y}^{*}(t)-F(t)\right)
\end{aligned}
$$

where $y^{*}(t)$ is the desired reference, and $k_{p}, k_{i}$ and $k_{d}$ are the equivalent proportional integral differential (PID) coefficients. The LUD which varies with time is obtained by an algebraic estimator.

In a short time interval, its estimation value is approximately equal [23]. Suppose that the time interval is marked as $L$. The estimation value $\hat{F}(t)$ is

$$
\hat{F}(t) \approx F(t-L)=y^{n}(t-L)-\alpha u(t-L)
$$

The value of parameter $\mathrm{L}$ will influence the estimation value. The entire iPID based on time delay shows in the Figure 1.

Assume that the order $\boldsymbol{n}$ is equal 2 and the error is defined as $e=y^{*}-y$.

Substituting equation (2) into (1), the error equation of the closed-loop system can be derived as follows:

$$
\ddot{e}(t)+k_{d} \dot{e}(t)+k_{p} e(t)+k_{i} \int e(t) d t=0
$$

The $k_{p}, k_{i}, k_{d}$ are treated as Hurwitz polynomial coefficients. According to the error equation, the performance of a close-loop system is designed by the Hurwitz theorem.

However, the established condition of error equation is that the estimation of $F(t)$ is accurately, and its estimation value can compensate a real value in the equation (1).

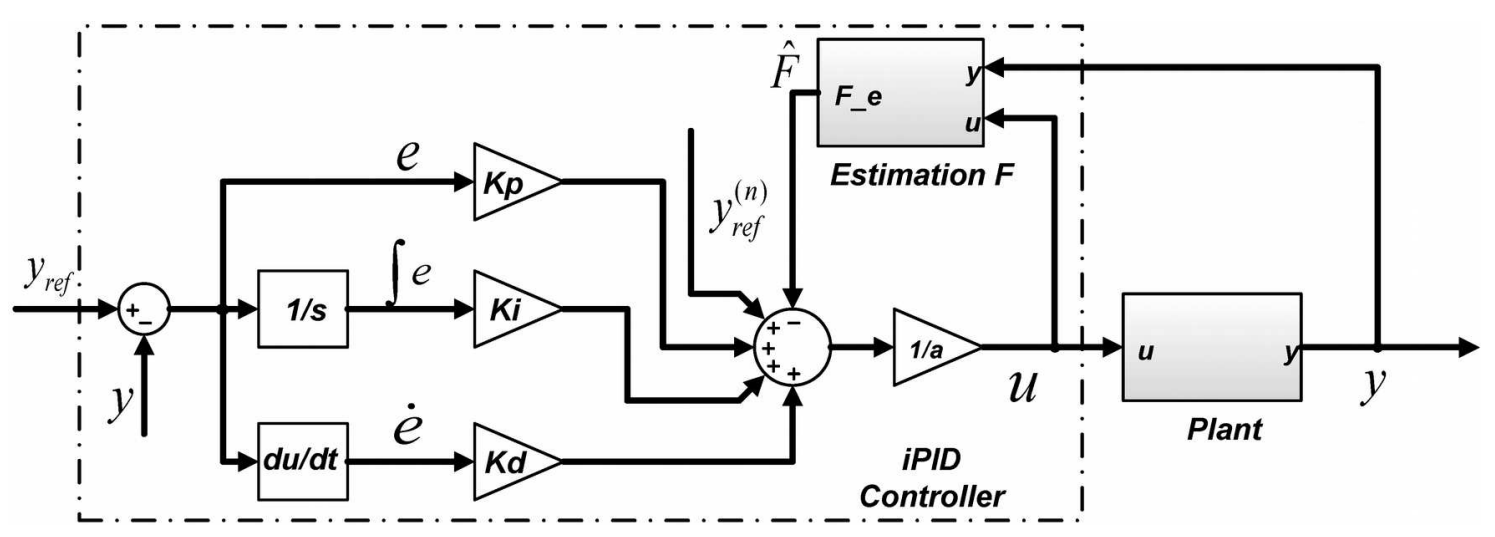

Figure 1. Structure of iPID control 
The estimation of $F(t)$ is a crucial issue. The controller of performance is strongly depended on an observer. The ideal input is not easily to attain by the observer. In addition, if the estimation error and the uncertain parameter of $\alpha$ are considered, the input can be rewritten as

$$
\begin{aligned}
u(t) & =\frac{1}{\alpha_{0}}\left(k_{p} e(t)+k_{i} \int e(t) d t+\right. \\
& \left.+k_{d} \dot{e}(t)+\ddot{y}^{*}(t)-\hat{F}(t)\right)
\end{aligned}
$$

where $\alpha_{0}$ is the nominal value of $\alpha$. Substituting (5) to (1), the system model can be described as

$$
\begin{aligned}
\ddot{y}(t) & =F(t)+\alpha\left[\frac { 1 } { \alpha _ { 0 } } \left(k_{p} e(t)+k_{i} \int e(t) d t+\right.\right. \\
& \left.\left.+k_{d} \dot{e}(t)+\ddot{y}^{*}(t)-\hat{F}(t)\right)\right]
\end{aligned}
$$

Moreover, we can obtain the error equation

$$
\ddot{e}(t)+k_{d} \dot{e}(t)+k_{p} e(t)+k_{i} \int e(t) d t=f(t)
$$

with

$$
f(t)=\left(\alpha / \alpha_{0}\right) \hat{F}(t)-F(t)+\left(\alpha / \alpha_{0}-1\right) \ddot{y}(t) .
$$

And $f$ is the error of disturbance and is estimated by an observer. The value of $f$ is related not only to the estimation error, but also to the parameter $\alpha$ and output. To solve this problem and improve the estimated precision, an auxiliary component is added to the input in the next section for compensating the estimation errors.

\section{Designing iPDNN Controller and Analysing Its Stability}

\subsection{Intelligent proportion differential neural network controller}

In this part, we will design a controller to compensate the unknown disturbance and reduce the candidate parameter. In order to reduce complexity of the controller, two terms of proportion integration differential are considered simply. If the ultra-local model is treated as one order $(n=1)$, the differential term is neglected. Here, we select proportion and differentiation (PD) parts as an example to elaborate iPDNN controller in detail. The architecture of iPDNN is shown in the Figure 2.

The extra input is defined as $u_{e}(t)$. The whole input of iPDNN control is

$$
u(t)=\frac{1}{\alpha_{0}}\left(k_{p} e(t)+k_{d} \dot{e}(t)+\ddot{y}^{*}(t)-\hat{F}(t) u_{e}(t)\right)
$$

Define a state vector $x=\left[\begin{array}{ll}x_{1} & x_{2}\end{array}\right]^{T}=\left[\begin{array}{ll}e & \dot{e}\end{array}\right]^{T}$. The error equation (7) is rewritten as statespace form, we have

$$
\dot{x}=A x+B \cdot f
$$

where $A=\left[\begin{array}{cc}0 & 1 \\ -k_{p} & -k_{d}\end{array}\right], \quad B=\left[\begin{array}{l}0 \\ 1\end{array}\right]$.

Suppose that the ideal approximation by neural network is $\hat{f}\left(x, w^{*}\right)$, it also satisfied $\max \left\|\hat{f}\left(x, w^{*}\right)-f\right\|<\varepsilon$. And $w^{*}$ is the optimal weights.

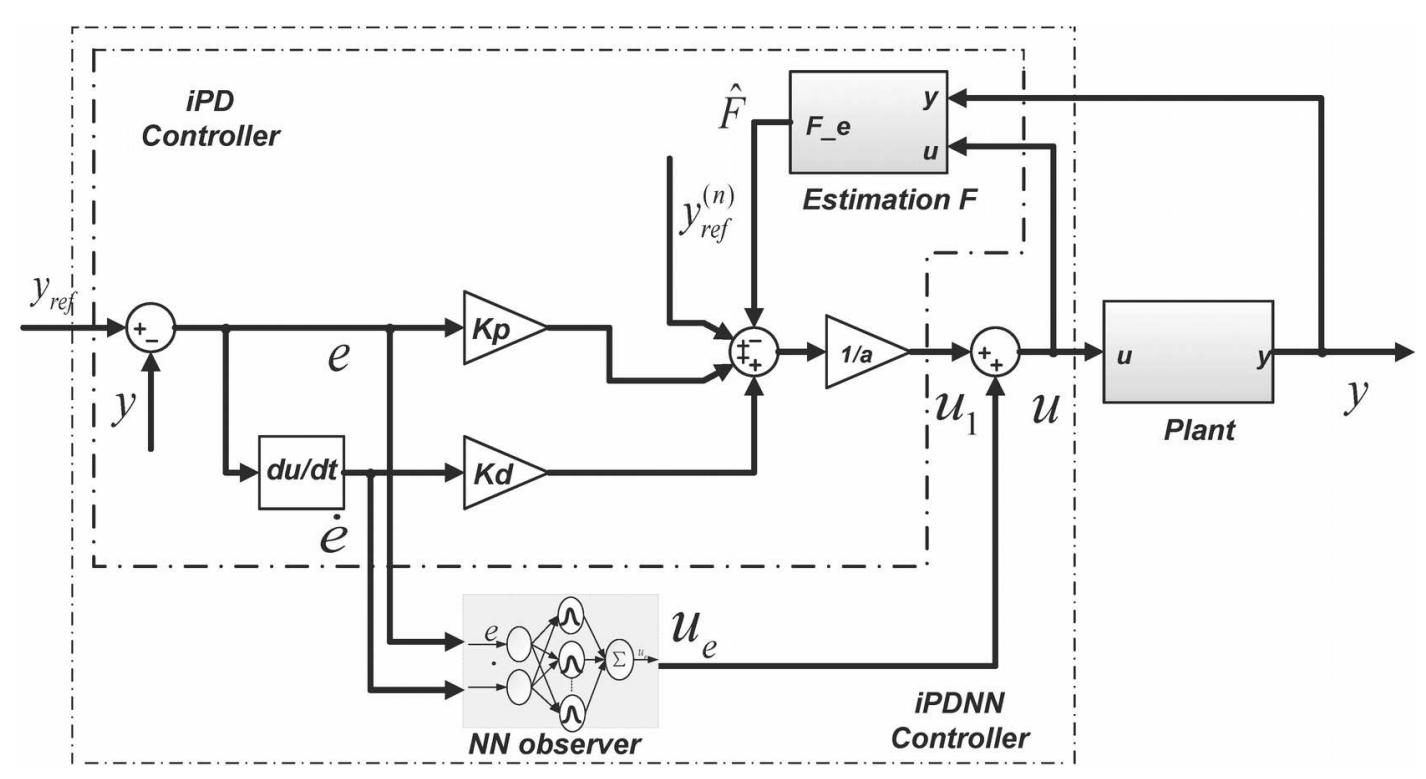

Figure 2. Structure of iPDNN control 
Considering the advantage of university approximation, a radial basis function (RBF) neural network is selected to approach the disturbance [24]. A Gaussian function for the hidden layer function is

$$
\varphi=\exp \left(\frac{\|x-c\|}{2 \sigma}\right)
$$

where $c=\left[\begin{array}{llll}c_{1} & c_{2} & \ldots & c_{n}\end{array}\right]$ is the center vector of the receptive field and $\sigma$ is the width of Gaussian function.

Hence, the ideal output of neural network for estimating the disturbance is

$$
\hat{f}\left(x, w^{*}\right)=w^{* T} \varphi
$$

Define $\quad \mu=f-\hat{f}\left(x, w^{*}\right)$ and substituting equation (15) to equation (13), we have

$$
\begin{aligned}
& \dot{x}=A x+B\left[f-\hat{f}\left(x, w^{*}\right)+\hat{f}\left(x, w^{*}\right)\right] \\
& \dot{x}=A x+B\left(\hat{f}\left(x, w^{*}\right)+\mu\right)
\end{aligned}
$$

The estimation of disturbance by direct RBF neural network is marked as

$$
\hat{f}(x, \hat{w})=\hat{w}^{T} \varphi
$$

where $\hat{w}$ is the neural network weight vector. In order to compensate the disturbance, the extra input $u_{e}$ is designed as

$$
u_{e}=-\hat{f}(x, \hat{w})
$$

The weight error is defined as $\widetilde{w}=w^{*}-\hat{w}$, the error equation can be simplified as

$$
\begin{aligned}
\dot{x} & =A x+B\left(\hat{f}\left(x, w^{*}\right)+u_{e}+\mu\right)= \\
& =A x+B\left(\widetilde{w}^{T} \varphi+\mu\right)
\end{aligned}
$$

Also,

$$
\dot{x}=A x+B\left(\widetilde{w}^{T} \varphi+\mu\right)
$$

\subsection{Stability of iPDNN control}

Assume that $P, Q$ is a symmetric positive definite matrix and satisfy the equation (18).

$A^{T} P+P^{T} A=-Q$

Then, a candidate Lyapunov function can be selected as

$$
V=\frac{1}{2} x^{T} P x+\frac{1}{2 \gamma} \widetilde{w}^{T} \widetilde{w}
$$

Take its derivative, we have

$$
\begin{aligned}
\dot{V} & =\frac{1}{2}\left(\dot{x}^{T} P x+x^{T} P \dot{x}\right)+\frac{1}{\gamma} \dot{\widetilde{w}}^{T} \widetilde{w}= \\
& =-\frac{1}{2} x^{T} Q x+\varphi^{T} \widetilde{w} B^{T} P x+ \\
& +\mu^{T} B^{T} P x-\frac{1}{\gamma} \dot{\hat{w}} \widetilde{w}
\end{aligned}
$$

Considering that $\widetilde{w} \in \mathfrak{R}^{n \times 1}, \quad \varphi \in \mathfrak{R}^{n \times 1}$, $B \in \mathfrak{R}^{2 \times 1}, \quad P \in \mathfrak{R}^{2 \times 2}$ and $x \in \mathfrak{R}^{2 \times 2}$, it is obvious that $B^{T} P x \in \mathfrak{R}, \quad \varphi^{T} \widetilde{w} \in \mathfrak{R}$, and $\varphi^{T} \widetilde{w} \cdot B^{T} P x=B^{T} P x \cdot \varphi^{T} \widetilde{w}$. Therefore, we have

$$
\begin{aligned}
\dot{V} & =-\frac{1}{2} x^{T} Q x+\left(B^{T} P x \varphi^{T}-\frac{1}{\gamma} \dot{\hat{w}}^{T}\right) \widetilde{w}+ \\
& +\mu^{T} B^{T} P x
\end{aligned}
$$

If the learning algorithms for weight is selected as

$$
\dot{\hat{w}}=\gamma B^{T} P x \varphi^{T}=\gamma \varphi x^{T} P B
$$

Cancelling the middle polynomial, we have

$\dot{V}=-\frac{1}{2} x^{T} Q x+\mu B^{T} P x$

Since $\|\mu\|=\left\|f-\hat{f}\left(x, w^{*}\right)\right\| \leq \mu_{0}, \quad\|B\|=1$ and $\|P x\| \leq \lambda_{\max }(P)\|x\|$, we have

$$
\begin{aligned}
\dot{V} & =-\frac{1}{2} x^{T} Q x+\mu B^{T} P x \leq \\
& \leq-\frac{1}{2} \lambda_{\text {min }}(Q)\|x\|^{2}+\mu_{0} \lambda_{\text {max }}(P)\|x\|= \\
& =-\frac{1}{2}\|x\|\left[\lambda_{\text {min }}(Q)\|x\|-2 \mu_{0} \lambda_{\text {max }}(P)\right]
\end{aligned}
$$

If $\lambda_{\min }(Q)\|x\|-2 \mu_{0} \lambda_{\max }(P)>0$, we obtain $\dot{V}<0$. Therefore, in the closed loop system, it is stable, if $\|x\|>2 \mu_{0} \lambda_{\max }(P) / \lambda_{\text {min }}(Q)$.

\section{Simulation Results}

In order to illustrate performance of the proposed method, an inverted pendulum plant which is illustrated in Figure 3 is employed and

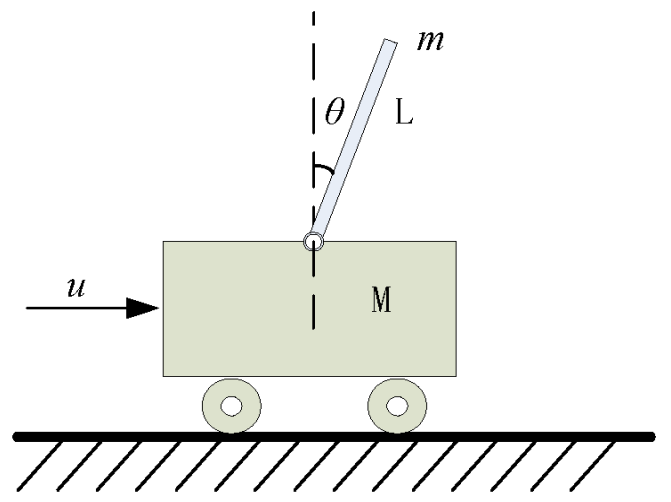

Figure 3. The inverted pendulum system 
tested under three different conditions. And according [25], this referred model is described as follows

$$
\begin{aligned}
& \dot{x_{1}}=x_{2} \\
& \dot{x_{2}}=a(x)+b(x) u(t)
\end{aligned}
$$

where

$$
\begin{aligned}
& b(x)=\frac{\cos x_{1}}{\frac{4}{3}(M+m) l-m l \cos ^{2} x_{1}}, \\
& a(x)=\frac{g \sin x_{1}-\frac{m l x_{2}^{2} \sin \left(2 x_{1}\right)}{2(M+m)}}{\frac{4}{3} l-\frac{m l \cos ^{2} x_{2}}{M+m}}
\end{aligned}
$$

and $x_{1}$ is the angle displacement whose unit is rad. $g=9.8 \mathrm{~m} / \mathrm{s}$ is the gravity acceleration coefficient, $M=1.0 \mathrm{~kg}$ and $m=0.1 \mathrm{~kg}$ are respectively the mass of cart and pole, $l=0.5 \mathrm{~m}$ is the length of the pole, and $u(t)$ is an input for the applied force control.

If we set the initial point in $x=[0.25,0]^{T}$, we have $\alpha_{0}=3 /(4 M l+m l)$. And $k_{p}=100$, $k_{d}=10, Q=[50,0 ; 0,50]^{T}, \gamma=10$.

\subsection{Case I - Balance point}

In order to demonstrate the performance, a direct adaptive neural network (NN) controller is applied to compare [25] with the proposed iPDNN controller to the balance point. Their corresponding simulation results are illustrated in Figures 4-6.The Figures 4-5 show the output of angular displacement and its error. The input is shown in the Figure 6.

Comparing with the NN method, both iPD and iPD methods have a good transient response from the response time. From the error curve in the Figure 5, the steady error of iPDNN control is less than the others. Furthermore, the extra controller by the RBF neural network can track the disturbance.

\subsection{Case II - Tracking performance of a sinusoidal signal}

In this case, we select a sinusoidal signal $\sin (\pi t / 6)$ as a reference. Their corresponding simulation results are shown in Figures 8-11. The values of parameters in controller are the same with the case I.

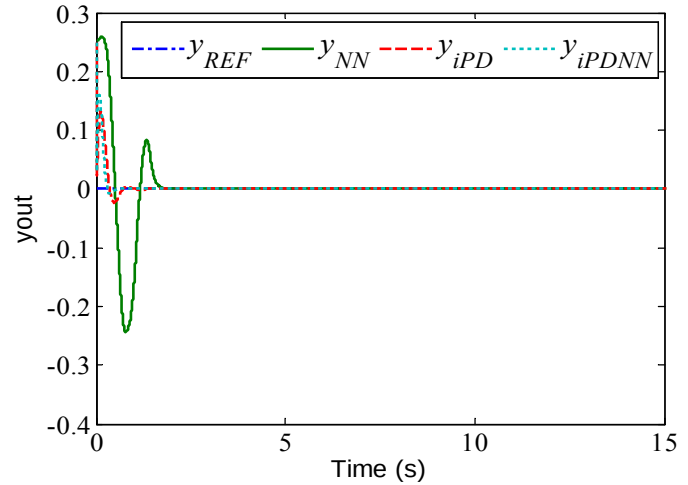

Figure 4. Output results withiPD, NN and IPDNN

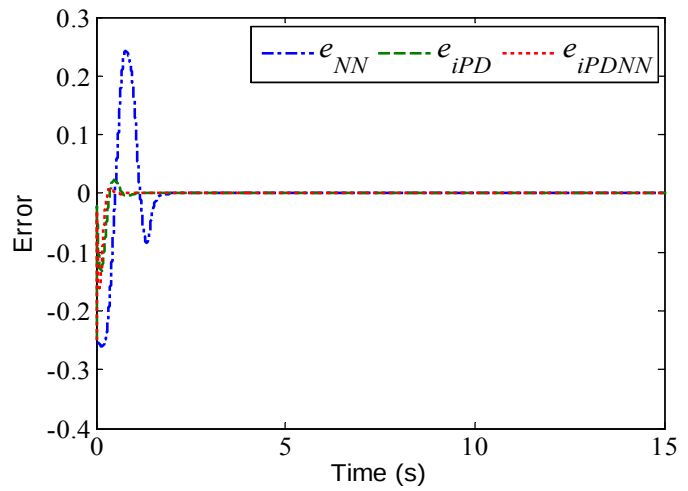

Figure 5. Errors of outputs withiPD, NN and iPDNN

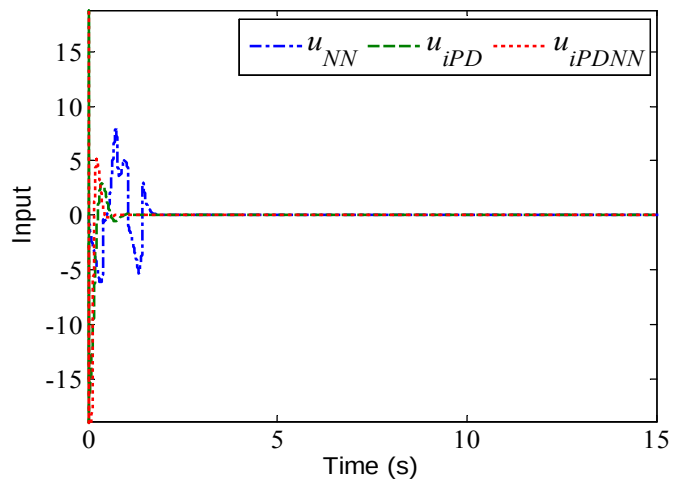

Figure 6. Control input withiPD, NN and iPDNN

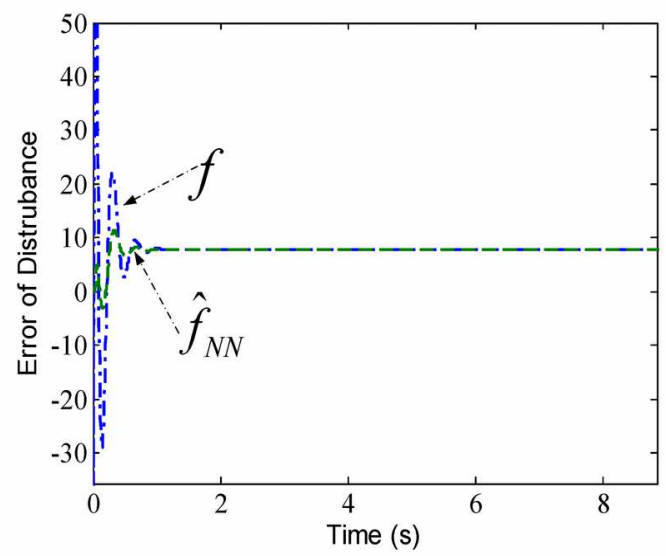

Figure 7. Estimation error of disturbance by Neural Network 
The Figures 8-9 show respectively the output of angular displacement and its error. The Figure 10 shows the output of controller. The Figure

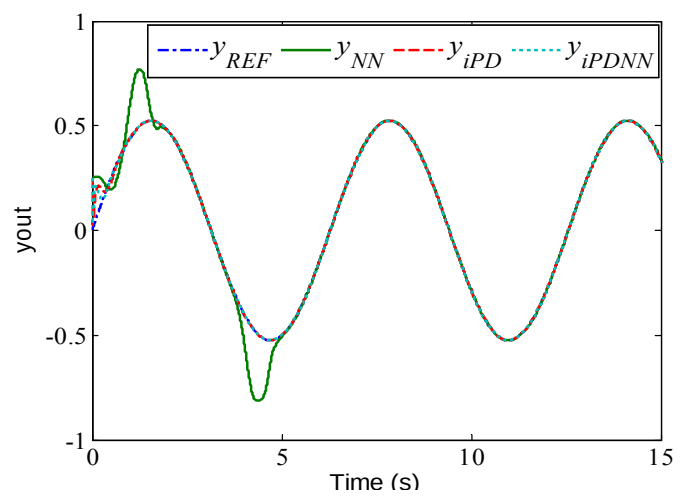

Figure 8. Output results with NN, iPDand iPDNN

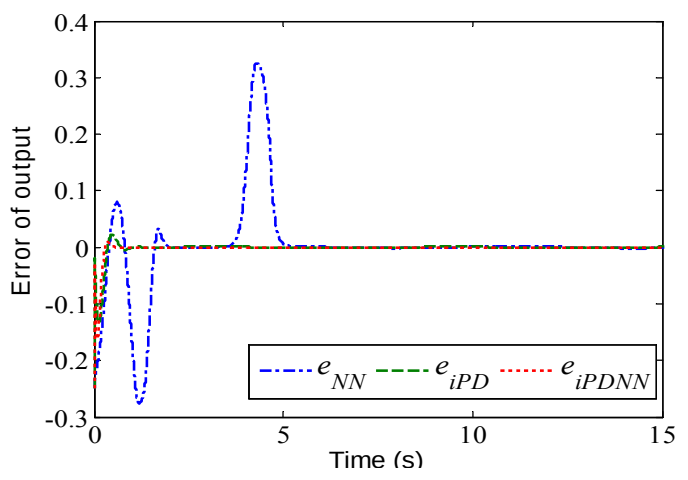

Figure 9. Errors of outputs with NN, iPD and iPDNN

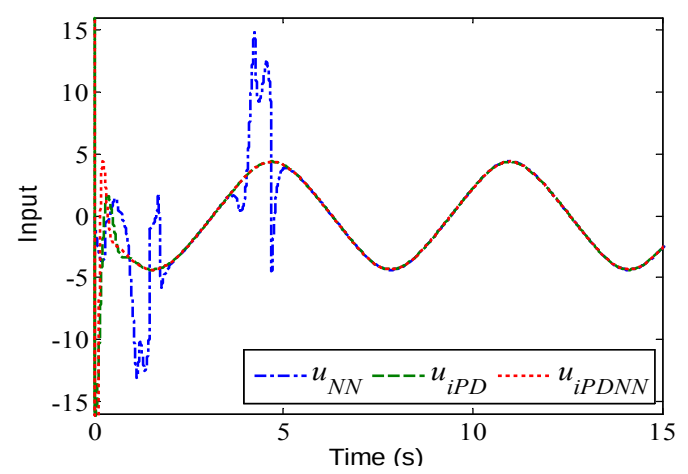

Figure 10. Inputs with NN, iPD and iPDNN

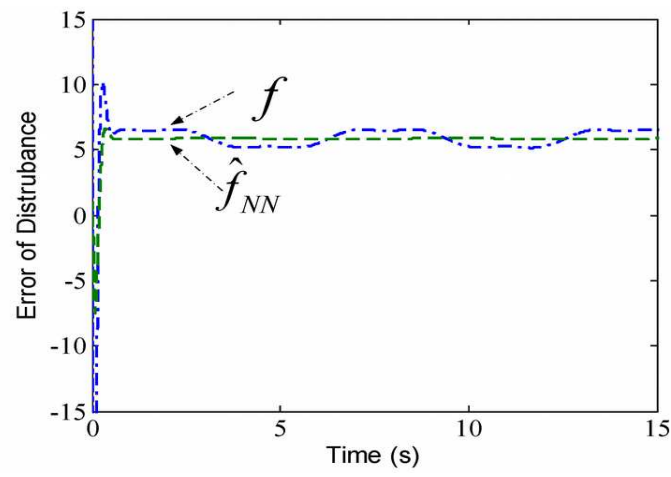

Figure 11. Estimation error of disturbance by Neural Network
11 shows the estimation error of disturbance by Neural Network. From these results, we can conclude that the proposed iPDNN controller can reduce its steady errors by comparing with the $\mathrm{NN}$ controller and iPD controller.

\subsection{Case III - Parameter variations of input gain and time interval}

In this case, the input gain $\alpha=20,15,10,8,5$, 2and time interval $L=0.0001,0.001,0.002$, 0.005 are selected. We also choose the sine signal $\sin (\pi t / 6)$ as a reference. Under these different conditions, the obtained simulation results are illustrated in Figures 12-13, which show respectively the errors of output under parameter variation of input gain and time interval. From these results, we can see that the iPDNN control maintains good robustness even under different parameter variation values.

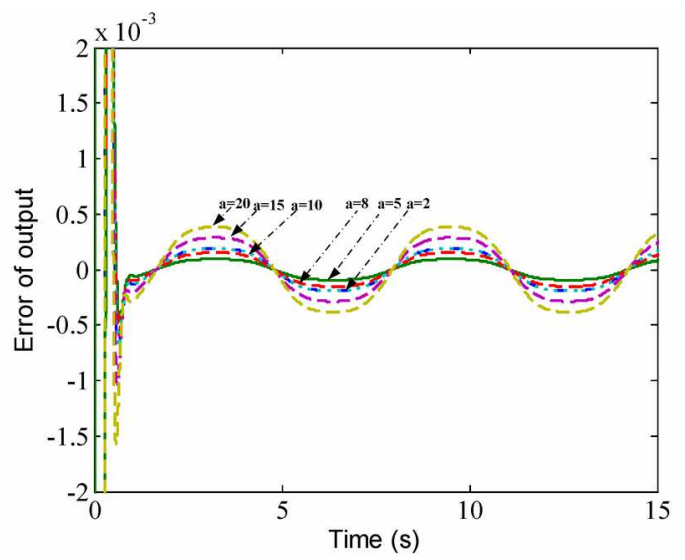

Figure 12. Errors of output by varying input-gain (a)

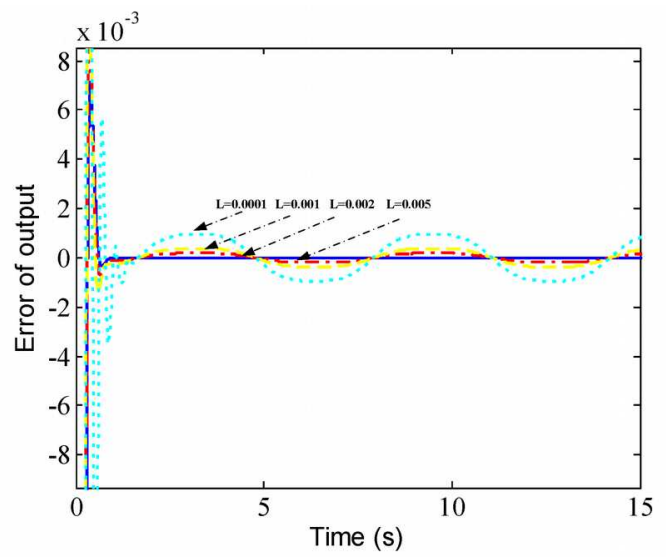

Figure 13. Errors of output by varying time interval $(\boldsymbol{L})$ 


\section{Conclusions}

In this paper, an intelligent Proportion Differential Neural Network (iPDNN) controller which is based on the iPID controller and RBF neural network is proposed. By adding an augmented neural network observer and compensating the estimation error, the iPDNN controller has effective ability of estimating the error of LUD and reduces the stable error in the closed-loop system. Also, it guarantees the stability of a closed-loop system. In order to demonstrate the performance of the proposed iPDNN controller, an inverted pendulum system has been tested on three different conditions and compared with methods of NN and iPD controller. Moreover, the proposed iPDNN controller maintains good robustness even under different parameter variation values.

\section{Acknowledgment}

This work is partially supported by the National Natural Science Foundation of China (61304077), by International Science \& Technology Cooperation Program of China (2015DFA01710), by the Natural Science Foundation of Jiangsu Province (BK20130765), and by the Chinese Ministry of Education Project of Humanities and Social Sciences (13YJCZH171), by the 11th Jiangsu Province Six talent peaks of high level talents (2014_ZBZZ_005), by the Jiangsu Province "Project Blue: young academic leaders" (2014) and by the Sino-French International collaboration project of CaiyuanPei (201404490146), and by the Funding of Jiangsu Innovation Program for Graduate Education (Grant No.KYLX_0377).

\section{REFERENCES}

1. BOLOURCHI, F., R. A. HESS, Nonlinear Model Reference Adaptive Control using Tap-delay Filters, IEEE Transactions on Systems, Man and Cybernetics, vol. 22, no. 2, 1992, pp. 360-368.

2. BOULKROUNE, A., M. M'SAAD, M. FARZA, Fuzzy Approximation-based Indirect Adaptive Controller for Multiinput Multi-output Non-affine Systems with Unknown Control Direction, IET
Control Theory \& Applications, vol. 6, no. 17, 2012, pp. 2619-2629.

3. CHANG, Y. W., Z. JING, Z. LIU, S. HONGYE, Robust Adaptive Control of Uncertain Nonlinear Systems in the Presence of Input Saturation and External Disturbance, IEEE Transactions on Automatic Control, vol. 56, no. 7, 2011, pp. $1672-1678$.

4. CHEN, W. L., L. YAN, Adaptive Dynamic Surface Control for Linear Multivariable Systems, Automatica, vol. 46, no. 10, 2010, pp. 1703-1711.

5. FLIESS, M., C. JOIN, Intelligent PID Controllers. 6th Mediterranean Conference on Control and Automation, 2008, pp. 326-331.

6. FLIESS, M., C. JOIN, Model-free Control, International Journal of Control, vol. 86 , no. 12 , 2013, pp. 2228-2252.

7. HAO, X. J., P. A. IOANNOU, Robust Adaptive Control for a Class of MIMO Nonlinear Systems with Guaranteed Error Bounds, IEEE Trans.on Automatic Control, vol. 48, no. 5, 2003, pp. 728-742.

8. HOJATI M., S. GAZOR, Hybrid Adaptive Fuzzy Identification and Control of Nonlinear Systems, IEEE Trans. on Fuzzy Systems, vol. 10, no. 2, 2002, pp. 198-210.

9. HSU, C.-F., Adaptive PI Hermite Neural Control for MIMO Uncertain Nonlinear Systems, Applied Soft Computing, vol. 13, no. 5, 2013, pp. 2569-2576.

10. HSU, C.-F. C.-M. LIN, R.-G. YEH, Supervisory Adaptive Dynamic RBFbased Neural-fuzzy Control System Design for Unknown Nonlinear Systems, Applied Soft Computing, vol. 13, no. 4, 2013, pp. 1620-1626.

11. HYEONGCHEOL L., M. TOMIZUKA, Robust Adaptive Control using a Universal Approximator for SISO Nonlinear Systems, IEEE Trans. on Fuzzy Systems, vol. 8, no. 1, 2000, pp. 95-106.

12. JOIN, C., F. CHAXEL, M. FLIESS, "Intelligent" Controllers on Cheap and Small Programmable Devices, 2013 Conference on Control and Fault-Tolerant Systems, 2013, pp. 554-559. 
13. LAFONT, F., J.-F. BALMAT, N. PESSEL, M. FLIESS, A Model-free Control Strategy for an Experimental Greenhouse with an Application to Fault Accommodation, Computers and Electronics in Agriculture, vol. 110, 2015, pp. 139-149.

14. LUNGU, M., R. LUNGU, C. ROTARU, New Systems for Identification, Estimation and Adaptive Control of the Aircrafts Movement, Studies in Informatics and Control, vol. 20, no. 3, 2011, pp. 273-284.

15. LUSU, G., L. PARSA, Model Reference Adaptive Control of Five-Phase IPM Motors Based on Neural Network, IEEE Transactions on Industrial Electronics, vol. 59, no. 3, 2012, pp. 1500-1508.

16. MENHOUR, L., B. D'ANDRÉA-NOVEL, M. FLIESS, D. GRUYER, H. MOUNIER, A New Model-free Design for Vehicle Control and Its Validation through an Advanced Simulation Platform, 14th European Control Conference (ECC), 2015, pp. 1-6.

17. MICHEL, L., C. JOIN, M. FLIESS, P. SICARD, AND A. CHERITI, Model-free Control of DC/DC Converters. 12th IEEE Workshop on Control and Modelling for Power Electronics (COMPEL), 2010, pp. 1-8.

18. PRECUP, R. E., RADAC, M. B., DRAGOS, C. A., PREITL, S., PETRIU, E. M., Model-free Tuning Solution for Sliding Mode Control of Servo Systems. 2014 8th Annual IEEE Systems Conference (SysCon), 2014, pp. 30-35.

19. SAN. S. G., J. WANG, Robust Adaptive Neural Control for a Class of Perturbed
Strict Feedback Nonlinear Systems, IEEE Transactions on Neural Networks, vol. 13, no. 6, 2002, pp. 1409-1419.

20. SCHAFER, R. W., What is a SavitzkyGolay Filter?, IEEE Signal Processing Magazine,, vol. 28(4), 2011, pp. 111-117.

21. VOJTESEK, J., P. DOSTAL, Simulation of Adaptive LQ Control of Nonlinear Process, Studies in Informatics and Control, vol. 21, no. 3, 2012, pp. 315-324.

22. WANG, H., Y. TIAN, D. ZHENG, ESObased iPI Common Rail Pressure Control of High Pressure Common Rail Injection System, Studies in Informatics and Control, vol. 25(3), 2016, pp. 273-282.

23. YOUCEF, T. K., O. ITO, A Time Delay Controller for Systems With Unknown Dynamics, Journal of Dynamic Systems, Measurement, and Control, vol. 112, no. 1, 1990, pp. 133-142.

24. YADMELLAT, P., S. K. Y. NIKRAVESH, Stabilizing Unstable Equilibria using Observer-based Neural Networks with Applications in Chaos Suppression. 2009 IEEE Symposium on Computational Intelligence in Control and Automation. 2009, pp. 96-103.

25. ZHANG, T., S. S. GE, C. C. HANG, Design and Performance Analysis of a Direct Adaptive Controller for Nonlinear Systems, Automatica, vol. 35, no. 11, 1999, pp. 1809-1817.

26. BOUSHAKI, R. Z., B. CHETATE, Y. ZAMOUM, Artificial Neural Network Control of the Recycle Compression System,Studies in Informatics and Control, vol. 23(1), 2014, pp. 65-76. 